\title{
Serum leptin levels in gastric cancer patients and the relationship with insulin resistance
}

\author{
Huseyin Begenik ${ }^{1}$, Mehmet Aslan¹, Ahmet Cumhur Dulger ${ }^{2}$, Habib Emre ${ }^{1}$, Ahu Kemik³, \\ Ozgur Kemik ${ }^{4}$, Ramazan Esen ${ }^{1}$
}

\begin{abstract}
${ }^{1}$ Department of Internal Medicine, Medical Faculty, Yuzuncu Yil University, Van, Turkey ${ }^{2}$ Department of Gastroenterology, Medical Faculty, Yuzuncu Yil University, Van, Turkey ${ }^{3}$ Department of Biochemistry, Medical Faculty, Istanbul University, Istanbul, Turkey ${ }^{4}$ Department of General Surgery, Medical Faculty, Yuzuncu Yil University, Van, Turkey
\end{abstract}

Submitted: 14 March 2013

Accepted: 12 April 2013

Arch Med Sci 2015; 11, 2: 346-352

DOI: 10.5114 /aoms.2015.50967

Copyright (c) 2015 Termedia \& Banach

\author{
Corresponding author: \\ Dr. Huseyin Begenik \\ Department \\ of Internal Medicine \\ Medical Faculty \\ Yuzuncu Yil University \\ Van, Turkey \\ Phone: +90 (432) 2150473 \\ Fax: +90 (432) 2167519 \\ E-mail: hbegenik@gmail.com
}

\section{Abstract}

Introduction: Serum leptin levels have been examined in various cancers, with conflicting results. However, there is limited information regarding serum leptin levels and insulin resistance in gastric cancer patients. Therefore, we aimed to investigate serum leptin levels, performance status, insulin levels and insulin resistance in patients with gastric cancer. In addition, we examined the relationship between these measurements and leptin levels. Material and methods: Thirty-nine patients with gastric cancer and 30 control subjects were enrolled in the study. Serum leptin, total protein, albumin, growth hormone, insulin and glucose levels were measured. The homeostasis model assessment (HOMA) was used to assess insulin resistance.

Results: Serum levels of insulin, glucose and growth hormone and insulin resistance were significantly lower in gastric cancer patients than controls ( $p<0.05$ for all). In the Pearson correlation analysis, insulin resistance was found to be significantly correlated with serum leptin levels in gastric cancer patients $(r=0.320, p=0.047)$. We observed a significant negative correlation between performance status and insulin resistance in patients with cachexia $(r=-0.512, p=0.030)$, while no association was found in non-cachectic patients.

Conclusions: We concluded that serum leptin levels are significantly lower in gastric cancer patients. In addition, gastric cancer patients have decreases in insulin levels, insulin resistance and growth hormone levels. This study found a positive association between serum leptin levels and insulin resistance. Moreover, there is a negative association between serum leptin levels and growth hormone levels. Thus, low insulin and growth hormone levels may suppress the production of leptin in gastric cancer patients.

Key words: leptin, insulin, insulin resistance, growth hormone, gender, gastric cancer.

\section{Introduction}

Gastric cancer is the second most common cause of cancer death worldwide. The prognosis for gastric cancer patients is poor in most industrialized countries, with a 5 -year survival rate of only $10 \%$. Gastric cancer is usually diagnosed at an advanced stage with limited curative options [1].

Leptin is a protein derived from the adipocyte family and is secreted into the bloodstream. Leptin is a member of the cytokine family and 
is an important signaling molecule in the energy regulation that stimulates appetite and weight gain [2]. Serum leptin levels correlate with body fat percentages, suggesting that leptin is an important signal of fat stores [2]. Initially, leptin was thought to be expressed and secreted only by adipocytes; however, their production in the placenta and in gastric, colorectal, and mammary epithelial tissues has been documented [3, 4]. Furthermore, leptin may act as a growth factor, participating in the development of cancer cell lines [5, 6]. Some studies have suggested that leptin may be involved in the functions of adipocytokines and in bone formation $[7,8]$.

Cancer cachexia, a cancer complication, is a complex and multifactorial syndrome that results from reduced food intake or metabolic abnormalities [9]. There is increasing evidence that the inflammatory response plays an important role in such alterations in gastrointestinal cancer patients [10]. Several inflammatory cytokines have been proposed as mediators of the cachectic process including tumor necrosis factor- $\alpha$ (TNF- $\alpha$ ), interleukin-1 (IL-1), interleukin-6 (IL-6), and interferon- $\alpha($ IFN- $\alpha)[11,12]$.

Although serum leptin levels have been extensively studied in some cancers, the results are conflicting. Some authors have reported decreased leptin levels in gastrointestinal cancer patients [13-16]. Others have reported increased leptin levels in breast, gastrointestinal and gynecologic cancer patients [17-19]. To our knowledge, there is only one report regarding serum leptin levels and insulin resistance in gastric cancer patients [20]. However, in that study, no association between serum leptin levels and insulin resistance was observed.

The aim of the present study was to investigate serum leptin levels, performance status, insulin levels and insulin resistance in patients with gastric cancer. In addition, we examined the relationship between these measurements and leptin levels.

\section{Material and methods}

\section{Subjects}

Thirty-nine patients with gastric cancer and 30 healthy controls were enrolled in this study.

The gastric cancer diagnosis was made through upper gastrointestinal endoscopic examinations and pathological findings. Staging of the gastric cancer was performed using both thorax and abdominal computed tomography (CT) on initial examination.

Ten of the tumors were well-differentiated, 15 were moderately differentiated and 14 were poorly differentiated gastric cancer. Patients were di- vided into three groups: 19 patients were stage I or IIA, 12 patients were stage IIB, and 8 were stage III.

The clinical parameters obtained in the study included age, gender, body mass index (BMI), cancer staging, cachexia status, body surface area and performance status.

None of the gastric cancer patients had received chemotherapy or radiotherapy. Patients with diabetes mellitus, hypertension, and renal or hepatic failure were excluded from the study. None of the gastric cancer patients were receiving antioxidant vitamin supplementation such as vitamin $\mathrm{E}$ or $\mathrm{C}$. In addition, the patients were not receiving any drugs and were not smoking or consuming alcohol.

The patient BMI was determined as the body weight divided by the square of the height $\left(\mathrm{kg} / \mathrm{m}^{2}\right)$. The patients with cancer were divided into two groups according to the presence (BMI $<20 \mathrm{~kg} / \mathrm{m}^{2}$ ) or absence $\left(\mathrm{BMI} \geq 20 \mathrm{~kg} / \mathrm{m}^{2}\right)$ of cachexia by using the BMI scale of Thomas et al. [21].

The patient performance status was evaluated using the World Health Organization (WHO) performance status guidelines.

The control group consisted of 30 healthy subjects with stable weight who were asymptomatic and had an unremarkable medical history and normal physical examination. None of the control subjects were receiving antioxidant vitamin supplementation such as vitamin $\mathrm{E}$ or $\mathrm{C}$. In addition, the subjects were not receiving any medication and were not smoking or consuming alcohol; the subjects had no known acute or chronic diseases.

The study protocol was performed in accordance with the Helsinki Declaration as revised in 2000. The study protocol was approved by the local ethics committee, and informed consent was obtained from each subject.

\section{Blood samples}

The patient blood samples were obtained in the morning following a 12 -h fasting period. The blood samples were collected into empty tubes and immediately stored at $4^{\circ} \mathrm{C}$. The serum samples were separated from cells by centrifugation at $3000 \mathrm{rpm}$ for $10 \mathrm{~min}$. Biochemical analyses were performed the same day. The serum samples were stored at $-80^{\circ} \mathrm{C}$ in plastic tubes until the leptin levels were analyzed.

\section{Measurement of serum leptin levels}

The serum leptin levels were measured as $\mathrm{ng} / \mathrm{ml}$ via a two-site immunoradiometric assay (IRMA) method by using the Human Leptin IRMA DSL-23100 kit (Diagnostic Systems Laboratories, Inc., Webster, Texas, USA). The measurements for the standards, controls, and sera were repeated 
for confirmation. The sensitivity of the test was $0.10 \mathrm{ng} / \mathrm{ml}$.

\section{Serum insulin levels}

Serum insulin levels were measured using an automated chemiluminescence autoanalyzer (Roche).

\section{Insulin resistance}

The insulin resistance index was calculated based on the fasting values for glycemia and insulinemia according to the homeostasis model assessment (HOMA) [22]. Insulin resistance (HOMA$\mathrm{IR})=$ fasting glycemia $(\mathrm{mmol} / \mathrm{l}) \times$ fasting serum insulinemia $(\mathrm{mlU} / \mathrm{ml}) / 22.5$.

\section{Other parameters}

The serum glucose, total protein and albumin levels were determined using commercially available assay kits (Abbott) with an autoanalyzer (Aeroset, Abbott).

\section{Statistical analysis}

The results are expressed as the mean \pm standard deviation. The nonparametric continuous variables were compared with the Mann-Whitney $U$-test. The parametric variables were compared using Student's $t$-test. Pearson's correlation analysis was used to determine the association between leptin levels, gender, age, BMI, serum albumin, total protein, growth hormone, and insulin and glucose levels. A linear regression analysis was used to determine the variables related to serum leptin levels. The results were considered to be significant when the $p$ value was less than 0.05 . The data were analyzed using the SPSS for Windows computer program (version 11.0).

\section{Results}

The demographic characteristics of the patients with gastric cancer and the control subjects are presented in Table I. There were no significant differences between gastric cancer patients and control subjects with respect to age or gender $(p>0.05)$. In addition, there were no significant differences between cachectic and non-cachectic patients with respect to age or gender $(p>0.05$; Table II).

The serum albumin levels were significantly lower in gastric cancer patients than control subjects $(p<0.05)$. However, there was no significant difference between gastric cancer patients and control subjects with respect to serum total protein levels ( $p>0.05$; Table $\mathrm{I})$. In addition, there were no significant differences between cachectic and non-cachectic patients with respect to serum total protein or albumin levels $(p>0.05)$.

Serum glucose levels were significantly higher in gastric cancer patients than controls $(p<0.05$; Table I); however, there was no significant difference in serum glucose levels between cachectic and non-cachectic patients $(p>0.05)$.

Serum leptin levels were significantly lower in gastric cancer patients than controls $(p<0.05$; Table II); however, there was no significant difference between cachectic and non-cachectic patients with respect to serum leptin levels $(p>0.05)$.

Table I. Demographic characteristics of gastric cancer and control subjects

\begin{tabular}{|c|c|c|c|}
\hline Parameter & Gastric cancer $(n=39)$ & Control $(n=30)$ & Value of $p$ \\
\hline Age [years] & $54 \pm 14$ & $51 \pm 9$ & NS \\
\hline Sex (female/male) & $23 / 16$ & $16 / 14$ & NS \\
\hline BMI $\left[\mathrm{kg} / \mathrm{m}^{2}\right]$ & $19.94 \pm 0.17$ & $20.60 \pm 0.98$ & NS \\
\hline Glucose [mg/dl] & $90.17 \pm 5.17$ & $83.96 \pm 5.28$ & $<0.05$ \\
\hline Total protein [g/dl] & $6.24 \pm 1.01$ & $6.57 \pm 0.51$ & NS \\
\hline Albumin $[\mathrm{g} / \mathrm{dl}]$ & $3.31 \pm 0.81$ & $4.16 \pm 0.50$ & $<0.05$ \\
\hline
\end{tabular}

Values are mean $\pm S D, B M I-$ body mass index, NS - non significant.

Table II. Hormone levels of gastric cancer and control subjects

\begin{tabular}{|c|c|c|c|}
\hline Parameter & Gastric cancer $(n=39)$ & Control $(n=30)$ & Value of $p$ \\
\hline Leptin [ng/ml] & $3.78 \pm 2.74$ & $9.03 \pm 2.01$ & $<0.05$ \\
\hline $\mathrm{GH}[\mathrm{ng} / \mathrm{ml}]$ & $2.74 \pm 1.17$ & $5.86 \pm 1.69$ & $<0.05$ \\
\hline Insulin $[\mu \mid \mathrm{U} / \mathrm{ml}]$ & $6.98 \pm 4.89$ & $11.10 \pm 2.57$ & $<0.05$ \\
\hline HOMA-IR & $1.57 \pm 1.13$ & $2.30 \pm 0.59$ & $<0.05$ \\
\hline
\end{tabular}

Values are mean $\pm S D, G H$ - growth hormone, HOMA-IR-homeostasis model assessment-insulin resistance. 
Serum insulin levels were significantly lower in gastric cancer patients than controls $(p<0.05$; Table II); however, there was no significant difference between cachectic and non-cachectic patients with respect to serum insulin levels $(p>0.05)$.

Serum growth hormone levels were significantly lower in gastric cancer patients than controls ( $p<0.05$; Table II); however, there was no significant difference between cachectic and non-cachectic patients with respect to serum growth hormone levels $(p>0.05)$.

Insulin resistance was significantly lower in gastric cancer patients than controls $(p<0.05$; Table II); however, there was no significant difference between cachectic and non-cachectic patients with respect to insulin resistance $(p>0.05)$.

Female subjects had higher serum leptin levels than male subjects in both the gastric cancer patients and in the control group, but these differences were not significant $(p>0.05)$.

We observed a negative association between performance status and insulin resistance in cachectic patients $(r=-0.512, p<0.030)$, while no such association was found in non-cachectic patients.

The serum leptin levels were found to be significantly correlated with insulin resistance in gastric cancer patients $(r=0.320, p=0.047)$ (Figure 1). In addition, leptin levels were found to be significantly correlated with serum growth hormone levels $(r=-0.327, p=0.042)$ (Figure 2) and insulin levels $(r=0.353, p=0.028)$ in gastric cancer patients.

A linear regression analysis was performed to identify factors that may exert an independent influence on leptin levels. Gender, age, BMI, serum albumin, total protein, growth hormone, insulin and glucose were included as independent variables. No relationship was found between serum leptin concentrations and these parameters in the cancer or control groups in a linear regression analysis $(p>0.05)$.

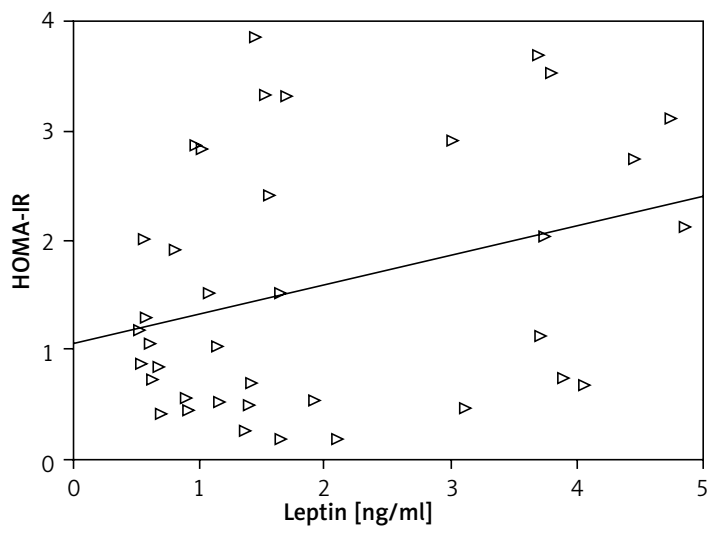

Figure 1. Correlation between serum leptin levels and insulin resistance in patients with gastric cancer $(r=0.320, p=0.047)$

\section{Discussion}

In the present study, we studied serum levels of leptin, insulin and growth hormone in gastric cancer patients. We also determined insulin resistance by using HOMA. In addition, we examined the associations among the serum leptin levels, insulin resistance and growth hormone levels in patients with gastric cancer. We found that gastric cancer patients had significantly lower serum leptin levels compared to healthy controls. We also observed significantly lower serum growth hormone and insulin levels in patients with gastric cancer than in healthy controls. In addition, we observed that insulin resistance was significantly lower in patients with gastric cancer than controls. Moreover, we found a significant positive association between serum leptin levels and insulin resistance in patients with gastric cancer. To our knowledge, this is the first report to describe the association between serum leptin levels and insulin resistance in gastric cancer patients.

Leptin is well known to play an important role in the regulation of food intake and energy balance [23]. Leptin controls body mass and metabolism by affecting the metabolic, neuroendocrine, reproductive and hematopoietic systems [24]. In cancer, there is regulatory dysfunction in the metabolic and neuroendocrine system, among others. Moreover, leptin has been shown to regulate cell proliferation in diverse normal and malignant tissues and to stimulate the proliferation of certain normal hematopoietic and epithelial cells. Leptin has been shown to promote the invasiveness of premalignant colon cells in vitro [25], and leptin is abundantly produced in the gastric mucosa [26].

Serum leptin levels have previously been investigated in some cancers including esophagus (16), gastric (4), colorectal $(8)$, and breast $(6,19)$ with conflicting results. Some authors have reported decreased leptin levels in gastrointestinal cancer patients [13-16], while others have reported increased

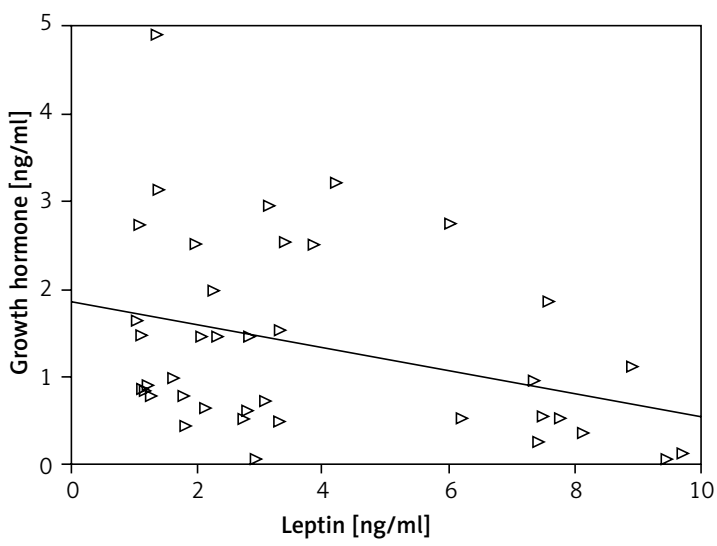

Figure 2. Correlation between serum leptin levels and serum growth hormone levels in patients with gastric cancer $(r=-0.327, p=0.042)$ 
leptin levels in breast, gastrointestinal cancer and gynecologic cancer patients [17-19]. Bolukbas et al. [15] observed significantly lower serum leptin levels in the gastrointestinal cancer group compared with controls. In addition, Nakajima et al. [16] observed that leptin levels were lower in patients with esophageal squamous cell carcinoma compared to controls. Bruun et al. [17] reported increased leptin levels in breast and gynecological cancer patients. Stattin et al. [18] observed that high serum leptin levels in patients correlated with an increased risk of colon, but not rectal, cancer. Tessitore et al. [19] reported that patients with breast cancer had higher serum leptin levels compared to controls. However, they did not observe an increase in serum leptin levels in patients with colorectal cancer compared to healthy controls.

There is only one report regarding serum leptin levels and insulin resistance in gastric cancer patients [20]. In that study, Kerem et al. [20] investigated serum leptin levels in gastric cancer patients. They reported that cachectic gastric cancer patients had significantly higher serum leptin levels than healthy controls and noncachectic gastric cancer patients. In that study, however, no significant differences in serum leptin levels between the non-cachectic and the healthy control groups were observed. In the same study, Kerem et al. [20] reported that insulin levels were increased in non-cachectic gastric cancer patients compared to cachectic patients; however, there was no significant difference between non-cachectic cancer patients and healthy subjects. They also reported that insulin resistance levels were not significantly different between the three groups. In the present study, with a Pearson correlation analysis, insulin resistance was found to be significantly correlated with serum leptin levels in gastric cancer patients.

Previous studies have demonstrated that serum leptin levels were significantly higher in women than in men with an equivalent BMI [27]. This sex-related difference could be explained by the fact that estrogens stimulate leptin production, whereas testosterone inhibits it [28]. In addition, circulating leptin levels are correlated with the BMI in healthy subjects [29]. Similarly, in the present study, serum leptin levels were higher in women than in men in gastric cancer patients and controls. However, this difference was not significant. A linear regression analysis has shown that serum leptin levels were not related to gender in either the cancer group or the controls. This observation may indicate that gender is not a determinant for serum leptin levels in cancer patients. As reported previously [27], other parameters such as BMI and age were not related to serum leptin levels in controls or cancer patients.
Epidemiologic studies have consistently shown that compared with nonwhite ethnic populations, people of European origin have a relatively low prevalence of insulin resistance [30]. Mente et al. [31] reported a significantly stronger correlation between serum insulin and leptin among European women than among South Asian women.

Cancer cachexia, characterized by marked weight loss, anorexia, nausea and general fatigue, is often associated with the presence or growth of a tumor and leads to malnutrition secondary to the induction of anorexia or decreased food intake [32]. Various mechanisms have been implicated in the etiology of cancer cachexia, and several studies have shown that cachexia associated with advanced cancer is associated with an increase in plasma cytokine levels [33]. Some authors have suggested that low leptin serum concentrations can be connected with the weight loss observed in cachectic cancer patients [15]. However, others did not confirm these observations, suggesting other unknown mechanisms of hypoleptinemia in those patients [19]. In addition, even after adjusting for the patient BMI or fat mass, the circulating leptin levels were significantly lower in cachectic patients than healthy controls [34]. Therefore, factors other than body fat mass may influence leptin production in cachexia.

In the present study, we did not observe any significant difference between cachectic and non-cachectic patients with respect to serum leptin levels. In addition, we did not observe any significant differences between cachectic and non-cachectic patients with regards to serum insulin or insulin resistance levels.

The patient performance status was evaluated using the World Health Organization performance status guidelines. The patient performance status was found to be an important parameter for determining the prognosis of patients with advanced cancer [35]. This scale is used by doctors and researchers to assess disease progression and patient daily living abilities and to determine patient prognosis and the appropriate treatment regimen. In the present study, we observed a negative association between performance status and insulin resistance in patients with cachexia, and no association was observed in non-cachectic patients. In addition, we observed that the performance status was positively correlated with cancer staging and negatively correlated with body surface area in esophagus cancer patients.

It has been shown that leptin secretion by the adipocyte is partly regulated by other hormones such as insulin, cortisol, growth hormone and testosterone [36]. Increasing evidence indicates that insulin can regulate leptin expression. Previous 
studies also suggest that the effect of insulin on leptin production is maintained by the permissive action of glucocorticoids [37]. Our results indicated that both cachectic and non-cachectic gastric cancer patients had low insulin levels compared to controls. Insulin, as an independent predictor, positively correlated with leptin levels. Therefore, chronic hypoinsulinemia may contribute to decreased leptin levels in gastric cancer patients. Increasing data provide evidence that leptin may also inhibit insulin synthesis and secretion [38]. Therefore, low leptin levels may contribute to the decreased insulin secretion in gastric cancer patients.

Growth hormone secretion declines with age and is affected by body composition. Growth hormone plays a crucial role in stimulating and controlling growth and metabolism but also has an important impact on body composition and fat distribution through its influence on energy metabolism and its lipolytic and nitrogen-sparing effects. Some, though not all $[39,40]$ studies found an inverse correlation between leptin and growth hormone in humans. Leptin is thought to accurately reflect fat mass and could therefore be a candidate protein that influences growth hormone secretion [39]. In the present study, the gastric cancer patients had significantly decreased growth hormone levels compared with the controls, which disagrees with previous findings that were based on healthy subjects [41, 42]. Huang et al. [42] indicate that growth hormone is a strong inhibitory mediator of leptin. They found that high growth hormone levels in gastric cancer patients may suppress leptin production. In the present study, in a Pearson correlation analysis, leptin levels were found to be significantly correlated with serum growth hormone levels and insulin levels.

In conclusion, we found that serum leptin levels are significantly lower in patients with gastric cancer. In addition, gastric cancer patients have decreased insulin levels, insulin resistance and growth hormone levels. In addition, the results from this study indicated a positive association between serum leptin levels and insulin resistance. Moreover, there is a negative association between serum leptin levels and growth hormone levels. Finally, low insulin and growth hormone levels may suppress the production of leptin in gastric cancer patients. These findings should be further evaluated in a larger population of gastric cancer patients.

\section{Acknowledgments}

The authors thank the staff at the Harran University Clinical Biochemistry for their generous and friendly assistance in every step of this study.

\section{Conflict of interest}

The authors declare no conflict of interest.

\section{References}

1. Klusek J, Głuszek S, Kozieł D. What is new in gastrointestinal cancer prevention - a review of the literature 2009-2010. Prz Gastroenterol 2011; 6: 78-84.

2. Stępień M, Wlazeł RN, Paradowski M, et al. Serum concentrations of adiponectin, leptin, resistin, ghrelin and insulin and their association with obesity indices in obese normo- and hypertensive patients - pilot study. Arch Med Sci 2012; 8: 431-6.

3. Ismail NA, Ragab S, Abd El Dayem SM, et al. Fetuin-A levels in obesity: differences in relation to metabolic syndrome and correlation with clinical and laboratory variables. Arch Med Sci 2012; 8: 826-33.

4. Hong SJ, Kwon KW, Kim SG, et al. Variation in expression of gastric leptin according to differentiation and growth pattern in gastric adenocarcinoma. Cytokine 2006; 33: 66-71.

5. Ollberding NJ, Kim Y, Shvetsov YB, et al. Prediagnostic leptin, adiponectin, C-reactive protein, and the risk of postmenopausal breast cancer. Cancer Prev Res (Phila) 2013; 6: 188-95.

6. Rose DP, Komninou D, Stephenson GD. Obesity, adipocytokines, and insulin resistance in breast cancer. Obes Rev 2004; 5: 153-65.

7. Housa D, Housová J, Vernerová Z, Haluzík M. Adipocytokines and cancer. Physiol Res 2006; 55: 233-44.

8. Kumor A, Daniel P, Pietruczuk M, Małecka-Panas E. Serum leptin, adiponectin, and resistin concentration in colorectal adenoma and carcinoma (CC) patients. Int J Colorectal Dis 2009; 24: 275-81.

9. Fearon KCH, Moses AGW. Cancer cachexia. Int J Cardiol 2002; 32: 73-81.

10. McMillan DC, Scott HR, Watson WS, Preston T, Milroy R, McArdle CS. Longitudinal study of body cell mass depletion and the inflammatory response in cancer patients. Nutr Cancer 1998; 31: 101-5.

11. Hood AF, Soter NA, Rappeport J, Gigli I. Graft-versus-host reaction. Cutaneous manifestations following bone marrow transplantation. Arch Dermatol 1977; 113: 1087-91.

12. Meisser A, Cameo P, Islami D, Campana A, Bischof P. Effects of interleukin-6 (IL-6) on cytotrophoblastic cells. Mol Hum Reprod 1999; 5: 1055-8.

13. Brown DR, Berkowitz DE, Breslow MJ. Weight loss is not associated with hyperleptinemia in humans with pancreatic cancer. J Clin Endocrinol Metab 2001; 86: 162-6.

14. Wallace AM, Kelly A, Sattar N, McArdle CS, McMillan DC. Circulating concentrations of "free" leptin in relation to fat mass and appetite in gastrointestinal cancer patients. Nutr Cancer 2002; 44: 157-60.

15. Bolukbas FF, Kilic H, Bolukbas C, et al. Serum leptin concentration and advanced gastrointestinal cancers: a case controlled study. BMC Cancer 2004; 4: 29-32.

16. Nakajima TE, Yamada Y, Hamano T, et al. Adipocytokines and squamous cell carcinoma of the esophagus. J Cancer Res Clin Oncol 2010; 136: 261-6.

17. Bruun JM, Lihn AS, Verdich C, et al. Regulation of adiponectin by adipose tissue-derived cytokines: in vivo and in vitro investigations in humans. Am J Physiol Endocrinol Metab 2003; 285: 527-33.

18. Stattin P, Lukanova A, Biessy C, et al. Obesity and colon cancer: does leptin provide a link? Int J Cancer 2004; 109: 149-52. 
19. Tessitore L, Vizio B, Jenkins O, et al. Leptin expression in colorectal and breast cancer patients. Int J Mol Med 2000; 5: 421-6.

20. Kerem M, Ferahkose Z, Yilmaz UT, et al. Adipokines and ghrelin in gastric cancer cachexia. World J Gastroenterol 2008; 14: 3633-41.

21. Thomas AE, McKay DA, Cutlip MB. A nomograph method for assessing body weight. Am J Clin Nutr 1976; 29: 302-4.

22. Matthews DR, Hosker JP, Rudenski AS, Naylor BA Treacher DF, Turner RC. Homeostasis model assessment: insulin resistance and beta-cell function from fasting plasma glucose and insulin concentrations in man. Diabetologia 1985 ; 28: 412-9.

23. Friedman JM, Halaas JL. Leptin and the regulation of body weight in mammals. Nature 1998; 395: 763-70.

24. Ganong WF. Review of medical physiology. 18th ed. Appleton and Lange, New York 1997; 223.

25. Attoub S, Noe V, Pirola L, et al. Leptin promotes invasiveness of kidney and colonic epithelial cells via phosphoinositide 3-kinase-, rho-, and rac-dependent signaling pathways. FASEB J 2000; 14: 2329-38.

26. Bado A, Levasseur S, Attoub $S$, et al. The stomach is a source of leptin. Nature 1998; 394: 790-3.

27. Stephens TW, Basinski M, Bristow PK, et al. The role of neuropeptide $Y$ in the antiobesity action of the obese gene product. Nature 1995; 377: 530-2.

28. Garofalo C, Surmacz E. Leptin and cancer. J Cell Physio 2006; 207: 12-22.

29. Considine RV, Sinha MK, Heiman ML, et al. Serum immunoreactive-leptin concentrations in normal weight and obese humans. N Engl J Med 1996; 334: 292-5.

30. Anand SS, Yusuf S, Vuksan V, et al. Differences in risk factors, atherosclerosis, and cardiovascular disease between ethnic groups in Canada: the Study of Health Assessment and Risk in Ethnic Groups (SHARE). Lancet 2000; 356: 279-84.

31. Mente A, Razak F, Blankenberg S, et al. Study of the Health Assessment And Risk Evaluation; Study of the Health Assessment And Risk Evaluation in Aboriginal Peoples Investigators. Ethnic variation in adiponectin and leptin levels and their association with adiposity and insulin resistance. Diabetes Care 2010; 33: 1629-34.

32. Calle EE, Thun MJ. Obesity and cancer. Oncogene 2004 23: 6365-78

33. Li WG, Gavrila D, Liu X, et al. Ghrelin inhibits proinflammatory responses and nuclear factor-kappa $B$ activation in human endothelial cells. Circulation 2004; 109: 2221-6.

34. Wallace AM, Kelly A, Sattar N, McArdle CS, McMillan DC. Circulating concentrations of "free" leptin in relation to fat mass and appetite in gastrointestinal cancer patients. Nutr Cancer 2002; 44: 157-60.

35. Forrest LM, McMillan DC, McArdle CS, Angerson WJ, Dunlop DJ. Evaluation of cumulative prognostic scores based on the systemic inflammatory response in patients with inoperable non-small-cell lung cancer. Br J Cancer 2003; 89: 1028-30.

36. Somasundar P, McFadden DW, Hileman SM, Vona-Davis L Leptin is a growth factor in cancer. J Surg Res 2004; 116: 337-49.

37. Saladin R, De Vos P, Guerre-Millo M, et al. Transient increase in obese gene expression after food intake or insulin administration. Nature 1995; 377: 527-9.

38. Seufert J, Kieffer TJ, Leech CA, et al. Leptin suppression of insulin secretion and gene expression in human pancreatic islets: implications for the development of adipogenic diabetes mellitus. J Clin Endocrinol Metab 1999; 84: 670-6.
39. Gill MS, Toogood AA, O'Neill PA, et al. Relationship between growth hormone $(\mathrm{GH})$ status, serum leptin and body composition in healthy and $\mathrm{GH}$ deficient elderly subjects. Clin Endocrinol 1997; 47: 161-7.

40. Manglik S, Cobanov B, Flores G, Nadjafi R, Tayek JA Serum insulin but not leptin is associated with spontaneous and growth hormone (GH)-releasing hormone-stimulated $\mathrm{GH}$ secretion in normal volunteers with and without weight loss. Metabolism 1998; 47: 1127-33.

41. Ozata M, Dieguez C, Casanueva FF. The inhibition of growth hormone secretion presented in obesity is not mediated by the high leptin levels: a study in human leptin deficiency patients. J Clin Endocrinol Metab 2003; 88: 312-6.

42. Huang Q, Zhang X, Jiang ZW, Liu BZ, Li N, Li JS. Hypoleptinemia in gastric cancer patients: relation to body fat mass, insulin, and growth hormone. JPEN J Parenter Enteral Nutr 2005; 29: 229-35. 